\title{
Transition to CFC-free inhalers
}

M Green

$\mathrm{P}$ ressurised metered dose inhalers (pMDIs) have served asthma patients admirably for decades and have remained popular even after the introduction of other devices. Their reliance on chlorofluorocarbons (CFCs) to propel the drug is now recognised as an environmental disadvantage.

Growing concern over the effect CFCs have on the atmosphere led to the signing of the Montreal Protocol in 1987, which stated that they be phased out. From January 1995, the production of CFCs was banned in the European Union except for essential uses. The rest of the developed world followed a year later. An agreement in 1994 stated that there should be a few specific exemptions, these include the use of CFCs in pMDIs, but only until safe and economically feasible alternatives could be developed.

Although the quantity of CFCs in pMDIs may seem insignificant on a global scale, pMDIs now constitute their biggest use (about 98\%), in the UK and Europe since CFCs were banned from all non-essential applications. Globally, the Technical and Economic Assessment Panel of the United Nations Environmental Programme has recommended that most inhalers should be CFC-free by 2000 , and all by $2005 .{ }^{1}$ EU legislation on phasing out the existing medically exempt CFCs is close to completion. Draft UK regulations state that as soon as two CFC-free beclomethasone pMDIs, plus two other inhaled steroids are licensed, the existing pMDIs should be withdrawn. Similar action is recommended when two salbutamol products are available. The UK government has indicated that it is committed to phasing out CFCs from pMDIs by the end of 1999 . $^{2}$

Although novel asthma therapies, such as oral compounds and new nebulisers, are being developed most of the focus of transition will be on pMDIs that contain alternative propellants, i.e. hydrofluoroalkanes (HFAs). These propellants do not contain chlorine, the component of CFCs that destroys ozone, and have been shown to be safe in extensive testing. ${ }^{3}$ At the moment, there is only one CFC-free pMDI inhaler available in the UK; this contains salbutamol. The first CFC-free steroid pMDI is likely to become available soon, and the major pharmaceutical companies producing asthma products are working on a number of other CFC-free inhalers. Therefore, practices will probably need to start switching their patients over when these are available. The latest British Thoracic Society guidelines on asthma treatment point out that there is a pressing need to plan for the transition to CFC-free pMDIs. ${ }^{4}$

THE ROLE OF PMDIS IN ASTHMA TREATMENT In the UK, pMDIs are the cheapest type of device available and are often the method of choice for those patients able to use them. Switching all patients over to pMDI alternatives is neither clinically nor economically desirable. Not all patients can use dry powder inhalers (DPIs) effectively, as they do not all have sufficient inspiratory flow rate to get the drug into their lungs. DPIs are also more expensive and changing every patient to them could have a major impact on the cost of asthma treatment.

It is unlikely that the role of pMDIs will decrease in the future. It is widely accepted that asthma is still underdiagnosed, even though awareness of the condition continues to increase among the public, as well as health professionals. This implies that the number of people being treated appropriately will also increase. The promotion of asthma guidelines and rational therapeutic policies in hospital and general practice may also mean more pMDIs are prescribed.

Chronic obstructive pulmonary disease (COPD) is another respiratory disease where the role of pMDIs may increase. COPD is common, probably underdiagnosed and certainly under-treated. ${ }^{5}$ Guidelines for the management of this condition recommend a graded approach to therapy, with inhaled bronchodilators and a trial of inhaled steroids at an early stage. ${ }^{6}$

\section{THE TRANSITION PROCESS}

An overall strategy is imperative in order to guide health professionals and patients through the transition process. Such a strategy has not been finalised, but it is likely to involve national and local organisations, the pharmaceutical industry and patients. The Department of Health has acknowledged that the availability of pMDIs that contain CFCs cannot be guaranteed beyond the shortterm - and that the transition will have to be handled carefully with the health of the patient as the priority. ${ }^{2}$ The current government has not yet revealed its plans for transition. However, at a recent meeting, ${ }^{7}$ a DoH spokeswoman stated that the Department's plans would be determined by the EU regulations.

\section{IMPLICATIONS}

The transition raises issues for health professionals, patients and health care purchasers. It will be important for respiratory physicians and other specialists in the field to be fully informed at the outset so that information may cascade to all who care for those with asthma and COPD. This can be achieved by a variety of methods, such as symposia within public meetings and editorials in medical journals. ${ }^{89,10}$ All concerned should be reminded that the transition is not optional. It seems likely that all CFCcontaining inhalers will have been withdrawn by 2005 , and most before then. Health professionals and patients must be informed about why the change is occurring and it should be explained that change is inevitable on environmental grounds.

In terms of therapeutic efficacy, most of the products will not alter as a result of the transition. However, the efficacy of some will be altered and health professionals will need to be made aware of what the changes are likely to be. With the first reformulated product, HFA-salbutamol, there is a difference in the shape and weight of the inhaler, the impact of the spray on the oropharynx and the taste. There are no differences in dosing and therefore no adjustments are needed during the changeover of bronchodilators. However, in the case of at least one of the CFC-free steroid inhalers, the characteristics may differ
Malcolm Green

Consultant Physician

Royal Brompton Hospital, Sydney Street, London SW3 $6 N P$.

Date received: 16/12/97 Date accepted: 09/01/98

Asthma in Gen Pract 1998 6(1): $3-5$ 
markedly from the products already on the market. For example, one CFC-free inhaled steroid has a greater deposition of drug in the lung due to the considerably smaller aerosol particles. ${ }^{11}$ This may mean that patients have to alter their steroid dose.

Guidelines for the different products must address the question of whether health professionals should maintain or alter the dose when transferring a patient to a CFC-free inhaler. This will be a change in the ethos of how asthma should be treated. Until recently, it had been recommended that a patient's initial steroid dose should be increased until symptom control had been achieved, ${ }^{12}$ but there is a new emphasis that 'start high and step down' may be a more effective strategy. ${ }^{4}$ This enhances patient confidence by reducing symptoms faster and reduces the risk of acute episodes. It may also improve the the eventual outcome.

Patients will need to be reassured about the transition and prepared for it. Health professionals are well aware of problems of efficacy when some patients are changed from branded to generic products and this type of problem needs to be managed carefully.

Patients need to be assured that:

- the new inhalers are less damaging to the environment

- the treatment is as safe as before;

- the treatment is as effective as before;

- the device is similar;

- the change has been recommended by the doctor.

They should also be warned that the device may feel and taste different and that the dosing recommendations may change. Patients should be able to express concerns they may have before or after the change. The experience of change in other countries is encouraging. In one study of 89 patients switched to CFC-free beclomethasone, 83 per cent described the switch as very easy whilst only two per cent said it was difficult. ${ }^{13}$

Collaboration between health authorities, health professionals and the pharmaceutical industry is important if the transition is to be smooth. The pharmaceutical industry will have to work in conjunction with pharmacists to provide information on the new inhalers to ensure continued compliance.

\section{LOCAL IMPLEMENTATION}

Relevant information - and exactly how the transition is going to be implemented - needs discussion at a local level. Some areas will have a local multidisciplinary Asthma Task Force, consisting of GPs, practice nurses, pharmacists, paediatricians, respiratory physicians and specialist nurses. Local branches of patient support groups such as the National Asthma Campaign can also be involved.

Preparing such a group to tackle transition is likely to have widespread benefits. Common messages and materials can be produced which are locally relevant and give the patients continuity as they pass between consultant, GP, nurse and pharmacist. Changing most patients in a locality at around the same time is likely to reduce problems at the primary-secondary care interface and will avoid patients being given a new CFC-free inhaler, only to be given an old one again during a hospital appointment or vice versa. This can also minimise problems for pharmacists by reducing the period in which they have to dispense both types of pMDI concurrently. It may be necessary to consider brand-name prescribing for simplicity and to avoid confusion; in any case generic CFC-free pMDIs may not be available for some years.

A local timetable for change can be co-ordinated with local press and media. Patient material containing key facts can be prepared and distributed within an area, and examples are already available from the National Asthma Campaign. A campaign called 'Freedom 2000 ' by 3M Health Care, and a similar programme by Allen and Hanburys, provide primary care health professionals with standard letters and patient information to distribute when the transition starts. Over the coming months it is likely that other such transition materials will be produced.

In primary care the transition may involve:

- a patient letter issued with a repeat prescription;

- GP or nurse consultations with key points covered and recommendations made. This will also provide an opportunity for patients to discuss fears and concerns;

- follow-up (if necessary) to discuss difficulties or anxieties.

\section{A TIMETABLE FOR TRANSITION}

Doctors can already switch patients to a CFC-free salbutamol pMDI, and CFC-free corticosteroids are likely to be available soon. When CFC-free $\beta$ agonists and steroids are both available some changeovers may occur at once by environmentally concerned doctors or at patient request. There may not be widespread change until national or local strategies are in place. These need to be considered and set in place as soon as practicable.

\section{Conclusions}

Concern for the environment is growing, and as doctors we must play our part. pMDIs are the last products using CFCs, and this exemption will soon be withdrawn. New CFC-free inhalers are coming on the market now, and we should prepare for transition with agreed local strategies within a national framework

\section{References}

1. United Nations Environment Panel. Technology and Economic Assessment Panel. Part III: Update of the 1994 T.O.C. Aerosols report and the PMDI transition strategy. UNEP June 1996. 2. Gerald Malone MP, secretary of state for health. Hansard: written answers; 8th July 1996.

3. Leach C. Preclinical safety of propellant HFA-134a and Airomir $^{\circledR}$ (salbutamol sulphate in CFC-free system) pMDI. $\mathrm{Br} J$ Clin Pract 1995; 79: 10-2.

4. British Thoracic Society, National Asthma Campaign, Royal College of Physicians et al. The British Guidelines on Asthma Management 1995. Review and Position Statement. Thorax 1997; 52: S1-S21.

5. British Lung Foundation. The Lung Report. Lung disease: a shadow over the nation's health. British Lung Foundation: London 1996.

6. British Thoracic Society. Guidelines for the Management of Chronic Obstructive Pulmonary Disease. Thorax 1997: 52(5): S1-S32.

7. Royal Pharmaceutical Society of Great Britain (RPSGB) Meeting on Transition, Tuesday October 28th 1997.

8. Partridge $\mathrm{M}$ and Woodcock A. Metered dose inhalers free of chlorofluorocarbons. BMJ 1995; 310: 684-5. 
9. Partridge M. Metered-dose inhalers and CFCs; what respiratory physicians need to know. Respir Med 1994; 88: 645-7.

10. Woodcock A. CFCs and inhalers. Lancet 1994; 344: 812. 11. Thompson P. Evaluating the therapeutic airways response to inhaled steroids. Clinical implication of improved targeting of inhaled steroids to the airways. European Respiratory Society Symposium: Stockholm 1996

12. Global Initiative for Asthma (GINA). Global strategy for asthma management and prevention. National Heart, Lung and Blood Institute (NHLBI)/World Health Organisation Workshop Report 1993. National Institutes of Health, NHLBI, Publication no. 95-3659. January 1995.

13. Cline A and Amies M. Patient preference for CFC-free aerosol metered dose inhaler (PMDI) replacement for current beclomethasone diproprionate (BDP) CFC PMDI. Eur Resp J 1996; 9(23): 254s

\title{
The role of inhaled steroids in COPD: the search for evidence
}

\author{
J Haughney
}

$\mathrm{C}$ hronic obstructive pulmonary disease (COPD) has for a long time been the 'Cinderella' respiratory condition in general practice. GPs and practice nurses see many patients with COPD, whose management is less clear than for asthma, with its evidence-based guidelines and treatment protocols. One of the most controversial questions in COPD is the value of inhaled corticosteroids. This is due to a fundamental lack of understanding about the underlying nature of the condition and the lack of data on long-term treatment.

COPD is defined as a chronic, slowly progressive disorder characterised by airways obstruction which does not change markedly over several months. Impairment of lung function is largely fixed but is partially reversible by bronchodilator (or other) therapy. It is a general term that covers many conditions which are now recognised as sharing the same underlying lung problems, including chronic bronchitis and emphysema. Many patients may also have some elements of asthma, explaining why lung function may be partially reversible. In general practice, COPD mainly affects older age groups and most cases are caused by tobacco smoking. Current indications suggest that the management of COPD may be suboptimal and greater efforts are warranted on the part of the GP.

COPD causes significantly more mortality and morbidity than other causes of airflow limitation in adults. In 1992, there were 26,033 deaths attributed to COPD, chronic bronchitis and emphysema in England and Wales, which accounts for 6.4 per cent of all male and 3.9 per cent of all female deaths. ${ }^{1}$ As one of the most common conditions seen in GP surgeries in patients over the age of 55, COPD has significant implications for a GPs workload. It results in two to four times more consultations than angina.

THE RATIONALE FOR USING CORTICOSTEROIDS IN COPD Many GPs already use inhaled corticosteroids in addition to inhaled bronchodilators in COPD, but is any real benefit achieved? There are at least two possible reasons why COPD patients might respond to antiinflammatory treatment, despite the general acknowledgment that the loss of lung tissue elasticity is relatively fixed. Some asthma patients may be misdiagnosed as COPD. The British Thoracic Society COPD guidelines ${ }^{1}$ point out that the differentiation of severe COPD from chronic severe asthma can be difficult because some degree of reversibility (shown as improvement in $\mathrm{FEV}_{1}$ ) can be achieved in the vast majority of patients. A spirometric response is considered positive if the $\mathrm{FEV}_{1}$ increases by $200 \mathrm{ml}$ and $15 \%$ from the baseline value. The pathological changes of bronchial asthma in the large airways can co-exist with those of COPD, which predominantly affects the small airways. Patients with COPD who respond to steroids may have a degree of inflammation which may be component specific to the disease.

While the presence of inflammatory changes in the airways of patients with COPD provides a rationale for the use of corticosteroids, ${ }^{2}$ however, the association between these changes, lung function and the therapeutic response to corticosteroids has not yet been clearly established. Whether a response to $30 \mathrm{mg}$ of prednisolone over two weeks predicts an ongoing improvement with inhaled corticosteroid treatment is debatable and results are awaited from ongoing studies.,4

\section{THE VALUE OF INHALED STEROIDS IN COPD}

Several uncontrolled retrospective studies suggest that long-term oral corticosteroids may slow the decline in $\mathrm{FEV}_{1}$ in patients with COPD ${ }^{5,6}$ However, long-term use of oral steroids would not generally be recommended, because of the risk of systemic side-effects. Inhaled corticosteroids offer an option for achieving similar benefits with less systemic side-effects.

An early trial suggested an improvement in $\mathrm{FEV}_{1}$ and reduction in the decline in $\mathrm{FEV}_{1}$ over one year's treatment with inhaled beclomethasone. ${ }^{7}$ A two year study with inhaled budesonide ( $1600 \mathrm{mcg} /$ day) showed significant reduction in respiratory symptoms, with a halving of the median decline in $\mathrm{FEV}_{1}$ in a group of nonallergic patients with COPD $(30 \mathrm{ml} / \mathrm{yr}$ in the budesonide group, compared to $60 \mathrm{ml} / \mathrm{yr}$ in the placebo group). The number of patients withdrawing due to pulmonary problems was significantly higher in the placebo group. ${ }^{8}$

\section{ONGOING TRIALS IN COPD}

What is needed to clarify the position is data from longterm studies investigating the use of inhaled corticosteroids in COPD. Three major multicentre trials are currently underway - The European Respiratory Society Study in

\author{
John Haughney \\ General Practitioner and \\ Editorial Board Member \\ Alison Lea Medical \\ Centre, Calderwood, East \\ Kilbride G74 3BE. \\ Date received: 08/12/97 \\ Date accepted: 12/01/98 \\ Asthma in Gen Pract 1998 \\ 6(1):5-7.
}

